\title{
Interference of Quantum Channels
}

\author{
Daniel K. L. Oi \\ Centre for Quantum Computation, Department of Applied Mathematics and Theoretical Physics, \\ University of Cambridge, Wilberforce Road, Cambridge CB3 0WA, United Kingdom
}

(Dated: October 30, 2018)

\begin{abstract}
We show how interferometry can be used to characterise certain aspects of general quantum processes, and in particular, the coherence of completely positive maps. We derive a measure of coherent fidelity, the maximum interference visibility, and the closest unitary operator to a given physical process under this measure.
\end{abstract}

PACS numbers: 03.67.-a

\section{INTRODUCTION}

A key requirement of quantum information processing is the ability to transform states coherently $\mathbf{1}$. In general, quantum processes will be described by quantum channels, or Completely Positive (CP) maps [2]. A relevant question is what happens when different processes act simultaneously on a system. Surprisingly, knowledge of the individual quantum channels alone is insufficient to specify the action of the simultaneous operation of both maps [3]. An experimental determination of the interference of the two maps reveals additional information about the maps which is not taken into account in their individual descriptions and is a measure of their coherent properties. From this, we may define an operational definition of coherent fidelity between CP maps. Thus, interferometry can be used a tool to extract information inaccessible to conventional process tomography [4].

\section{INTERFEROMETRY}

Single particle interference (Fig. 1) displays the key elements of quantum mechanics: the superposition of indistinguishable paths, and the complementarity of certain observables. Interference is a consequence of the possibility of the particle taking both paths, and any process which tends to label the path of the particle will reduce

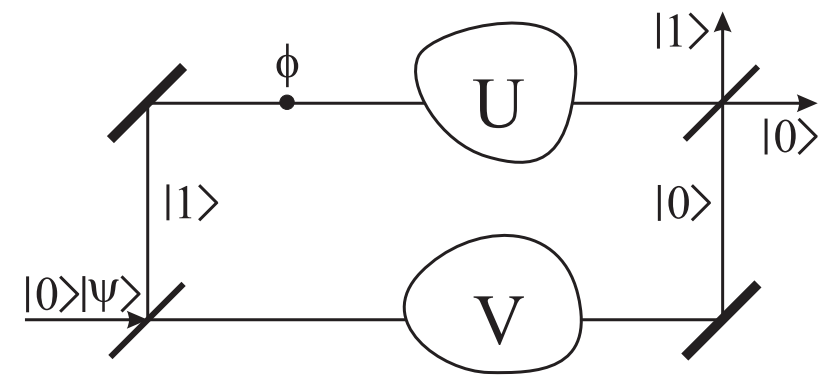

FIG. 1: Mach-Zender Interferometer. We allow the possibility of different quantum processes occurring in the upper and lower arms.

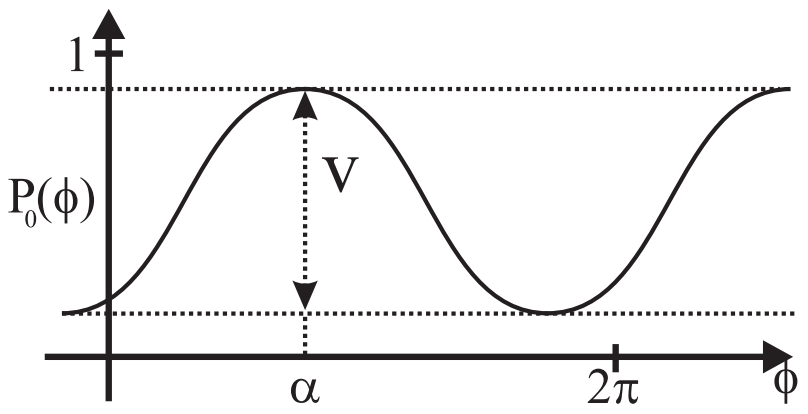

FIG. 2: Interference pattern showing a phase shift and reduction in visibility. The shift is a measure of the relative phase of the two quantum processes, and the reduction of visibility is a consequence of the leakage of path information into other degrees of freedom.

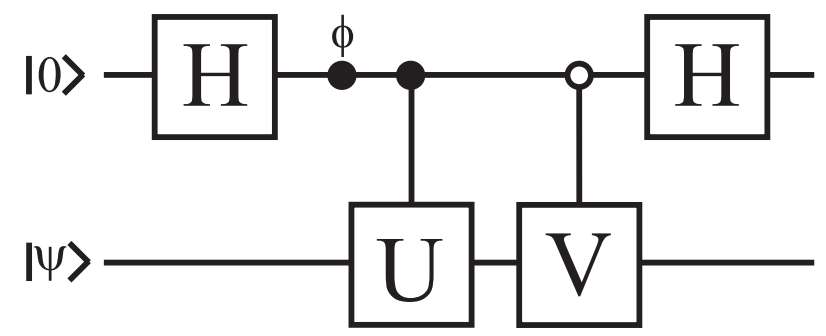

FIG. 3: Quantum Network for Interfering Unitaries. The actions of the unitary operations on the internal state of the particle (lower line) are controlled by the path of the particle (upper line).

the magnitude of the interference [ $\underline{5}$.

In general, the perfect interference pattern will be modified by the presence of quantum processes occurring in the upper and lower arms (Fig. 2). For unitary processes (Fig. 33), the evolution of a particle with initial internal state $|\psi\rangle \in \mathcal{H}_{d}$ is,

$$
\begin{aligned}
\left|\Psi_{i n}\right\rangle & =|0\rangle|\psi\rangle \\
& \mapsto \frac{1}{\sqrt{2}}(|0\rangle+|1\rangle)|\psi\rangle \\
& \mapsto \frac{1}{\sqrt{2}}\left(|0\rangle V|\psi\rangle+e^{i \phi}|1\rangle U|\psi\rangle\right)
\end{aligned}
$$




$$
\mapsto \frac{1}{2}\left[|0\rangle\left(V+e^{i \phi} U\right)|\psi\rangle+|1\rangle\left(V-e^{i \phi} U\right)|\psi\rangle\right] .
$$

The probability of finding the particle in the $|0\rangle$ state, corresponding to it exiting the interferometer from the horizontal output port, is given by

$$
\begin{aligned}
P_{0}(\phi) & =\frac{1}{4}\left\langle\psi\left|\left(V+e^{i \phi} U\right)^{\dagger}\left(V+e^{i \phi} U\right)\right| \psi\right\rangle \\
& =\frac{1}{2}(1+\mathrm{v} \cos (\phi-\alpha))
\end{aligned}
$$

where $|\mathrm{v}| \equiv\left|\left\langle\psi\left|U^{\dagger} V\right| \psi\right\rangle\right|$ is the new visibility of the interference pattern, and $\alpha \equiv \arg \left(\left\langle\psi\left|U^{\dagger} V\right| \psi\right\rangle\right)$ is the shift of the interference fringes [6]. The magnitude of the visibility is the fidelity of the states $U|\psi\rangle$ and $V|\psi\rangle$, i.e. the overlap of the states exiting the upper and lower arms of the interferometer [7]. The higher their fidelity - hence the lower their distinguishability - the greater the interference effect. Conversely, if the states exiting the upper and lower arms were perfectly distinguishable (orthogonal), there would be no interference.

If the initial state of the particle is $\varrho_{i n}=|0\rangle\langle 0| \otimes \rho$, the modified visibility is

$$
\mathrm{v} e^{i \alpha}=\operatorname{Tr}\left[\rho U^{\dagger} V\right]
$$

which is the expectation value $\left\langle U^{\dagger} V\right\rangle_{\rho}$. If we use the input $\rho=\frac{\pi}{d}$, the maximally mixed state (equivalent to randomly sampling over a uniform distribution of pure input states), then the visibility pattern gives us the quantity

$$
\operatorname{Tr}\left[U^{\dagger} V\right]=d \mathrm{v} e^{i \alpha},
$$

from which the Hilbert-Schmidt distance between two operators on $\mathcal{H}_{d}$ can be derived,

$$
\begin{aligned}
D^{2}(U, V) & =\operatorname{Tr}\left((U-V)^{\dagger}(U-V)\right) \\
& =2\left(d-\operatorname{Re}\left\{\operatorname{Tr}\left[U^{\dagger} V\right]\right\}\right) \\
& =2 d(1-\mathrm{v} \cos \alpha) .
\end{aligned}
$$

Hence, we have a direct estimate of the distance between unitary processes [8].

\section{CP MAPS}

The interference pattern can reveal important information in the case where the operations in the upper and lower arms are not unitary, but are $\mathrm{CP}$ maps $\mathcal{U}$ and $\mathcal{V}$. We will assume these are trace preserving and have the same input and output (finite dimensional) spaces. We can model this case by extending the state space of the entire system by appending two ancillas $\mathrm{F}$ and $\mathrm{E}$ (assuming that $\mathcal{U}$ and $\mathcal{V}$ are independent processes), which are coupled to the top and bottom paths by overall unitaries, $\mathbb{U}$ and $\mathbb{V}$, which implement the $\mathrm{CP}$ maps, $\mathcal{U}$ and $\mathcal{V}$ respectively [9] (Figs. 固\& 国),

$$
\begin{aligned}
& \mathcal{U}(\rho)=\operatorname{Tr}_{F}\left[\mathbb{U}\left(\rho \otimes\left|f_{0}\right\rangle\left\langle f_{0}\right|\right) \mathbb{U}^{\dagger}\right] \\
& \mathcal{V}(\rho)=\operatorname{Tr}_{E}\left[\mathbb{V}\left(\rho \otimes\left|e_{0}\right\rangle\left\langle e_{0}\right|\right) \mathbb{V}^{\dagger}\right],
\end{aligned}
$$

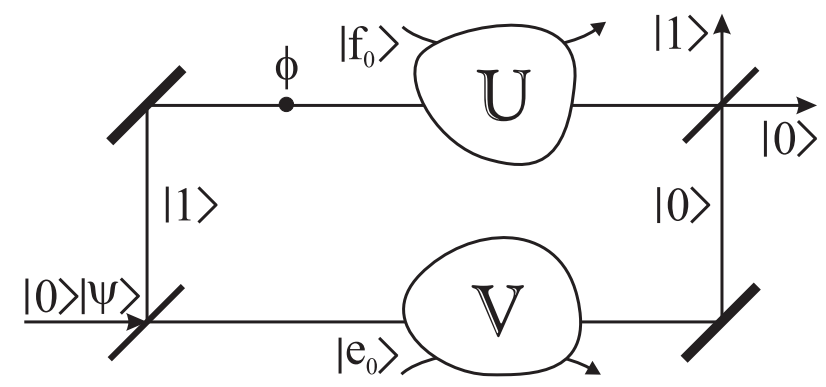

FIG. 4: Interference of two CP maps. We model the situation by implementing $\mathcal{U}$ and $\mathcal{V}$ by unitaries $\mathbb{U}$ and $\mathbb{V}$ respectively acting on larger Hilbert spaces, and then tracing out the ancillary systems.

where $\left\{\left|e_{\mu}\right\rangle\right\}$ and $\left\{\left|f_{\nu}\right\rangle\right\}$ are orthonormal bases and $\left|e_{0}\right\rangle$ and $\left|f_{0}\right\rangle$ are initial states of $\mathrm{E}$ and $\mathrm{F}$ respectively.

Note that for any CP map $\Lambda$, there exists many unitaries which implement $\Lambda$ and that we cannot distinguish by quantum process tomography the different instantiations. We may instead uniquely specify $\Lambda$ via the Jamiołkowski isomorphism [12],

$$
\Lambda \cong \varrho_{\Lambda} \equiv \mathbb{I} \otimes \Lambda\left(\left|\Psi_{+}\right\rangle\left\langle\Psi_{+}\right|\right),
$$

where $\left|\Psi_{+}\right\rangle$is the maximally entangled state and there exists a one-one correspondence between the set of $\mathrm{CP}$ maps and the set of bipartite density operators having one subsystem with maximally mixed reduced density operator.

We can trivially extend both the overall unitaries to act on the whole space of the particle, and ancillas $\mathrm{E}$ and F,

$$
\begin{aligned}
\tilde{\mathbb{U}} & =\mathbb{U} \otimes \mathbb{I}_{E} \\
\tilde{\mathbb{V}} & =\mathbb{V} \otimes \mathbb{I}_{F} .
\end{aligned}
$$

The action of the interferometer on an initially pure state of the particle is now given by

$$
\begin{aligned}
|\Psi\rangle & =|0\rangle|\psi\rangle\left|e_{0}\right\rangle\left|f_{0}\right\rangle \\
& \mapsto \frac{|0\rangle \tilde{\mathbb{V}}\left|\psi e_{0} f_{0}\right\rangle+e^{i \phi}|1\rangle \tilde{\mathbb{U}}\left|\psi e_{0} f_{0}\right\rangle}{\sqrt{2}} \\
& \mapsto \frac{\left(|0\rangle\left(\tilde{\mathbb{V}}+e^{i \phi \tilde{\mathbb{U}}}\right)+|1\rangle\left(\tilde{\mathbb{V}}-e^{i \phi \tilde{\mathbb{U}}}\right)\right)\left|\psi e_{0} f_{0}\right\rangle}{2} .
\end{aligned}
$$

Thus the probability of the particle exiting from the horizontal output port is given by

$$
\left.\mathrm{P}_{0}(\phi)=\frac{1}{4}\left|\left(\tilde{\mathbb{V}}+e^{i \phi} \tilde{\mathbb{U}}\right)\right| \psi e_{0} f_{0}\right\rangle\left.\right|^{2} .
$$

In general, if the particle has internal state $\rho$, the probability is

$$
\mathrm{P}_{0}(\phi)=\frac{1}{2}\left(1+\operatorname{Re}\left\{e^{i \phi} \operatorname{Tr}\left[\tilde{\mathbb{U}}^{\dagger} \tilde{\mathbb{V}} \rho \otimes\left|e_{0} f_{0}\right\rangle\left\langle e_{0} f_{0}\right|\right]\right\}\right) .
$$




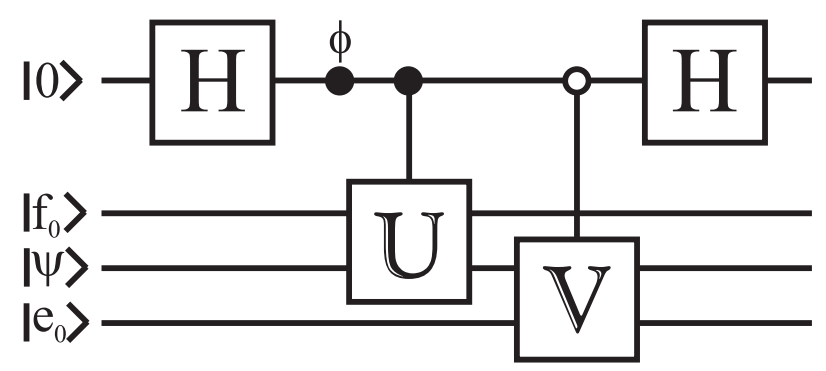

FIG. 5: Quantum network for the interference of two CP maps implemented by unitary operators acting upon the system and ancillas.

The relevant quantity in the above can be expressed as

$$
\operatorname{Tr}\left[\tilde{\mathbb{U}}^{\dagger} \tilde{\mathbb{V}} \varrho \otimes\left|e_{0} f_{0}\right\rangle\left\langle e_{0} f_{0}\right|\right]=\operatorname{Tr}\left[v_{0}^{\dagger} \nu_{0} \varrho\right],
$$

where the Kraus operators for $\mathcal{U}$ and $\mathcal{V}$ are given by

$$
\begin{aligned}
& \left\{v_{i}\right\}=\left\{\left\langle e_{i}|\mathbb{U}| e_{0}\right\rangle\right\} \\
& \left\{\nu_{j}\right\}=\left\{\left\langle f_{j}|\mathbb{V}| f_{0}\right\rangle\right\} .
\end{aligned}
$$

Again, if the input is the maximally mixed state, the interference pattern depends on $\frac{1}{d} \operatorname{Tr}\left[v_{0}^{\dagger} \nu_{0}\right]$ only. This reduces to the previously considered case if the operations in the upper and lower paths are unitary on the internal state of the particle.

It is interesting to note that the visibility is dependent on a particular decomposition of the two CP maps, in particular the overlap of the first Kraus operators of $\mathcal{U}$ and $\mathcal{V}$. We may interpret this in the framework of quantum jumps 10, 11]. The visibility is a consequence of the indistinguishability of the two possible paths of the particle through the interferometer, anything that serves to tag the passage of the particle serves to reduce the interference pattern. This may not just be an internal change in the state of the particle (created by differing unitary operations $U$ and $V$ ) but also any changes in the environment which may mark the particle's passage. Thus, the first Kraus operators of both $\mathcal{U}$ and $\mathcal{V}$ denote the action of the operation when there is no quantum jump of the environment "watching" the particle. The residual overlap concerns the "unitary" action of the CP map under this condition of no jump.

Note that even though a CP map may seem to be unitary when only acting on the internal degrees of freedom, i.e.

$$
\Lambda(\rho)=U \rho U^{\dagger},
$$

the first Kraus operator may be the zero operator, $\left\{\lambda_{0}=\right.$ $\left.0, \lambda_{1}=U\right\}$, and hence give zero visibility [17], e.g.

$$
\mathbb{U}\left(|\psi\rangle_{I}\left|f_{0}\right\rangle\right)=\left(U|\psi\rangle_{I}\right)\left|f_{1}\right\rangle \forall|\psi\rangle \in \mathcal{H}_{d}
$$

In this case, the map serves as an indicator of path, entangling the fact of the passage of the particle with the environment without altering the internal state. In the interferometer, this results in the destruction of all interference. In general, a CP map will necessarily entangle the passage of the particle with environmental degrees of freedom (a non-product $\mathbb{U}$ ), hence reducing the interference beyond the effect of altering the internal state of the particle.

We may thus define a coherent fidelity between two sets of Kraus operators implementing different CP maps as,

$$
\mathcal{F}\left(\left\{v_{i}\right\},\left\{\nu_{i}\right\}\right)=\frac{1}{d}\left|\operatorname{Tr}\left[v_{0}^{\dagger} \nu_{0}\right]\right|,
$$

and their relative phase,

$$
\mathcal{P}\left(\left\{v_{i}\right\},\left\{\nu_{i}\right\}\right)=\arg \left(\operatorname{Tr}\left[v_{0}^{\dagger} \nu_{0}\right]\right) .
$$

We may note that another fidelity measure on the set of CP maps has been defined via the Uhlmann fidelity 13. between the density operators defined in Eq. (8) [14],

$$
\mathcal{F}^{\prime}(\mathcal{U}, \mathcal{V})=\operatorname{Tr}\left[\sqrt{\sqrt{\rho_{\mathcal{U}}} \rho_{\mathcal{V}} \sqrt{\rho \mathcal{U}}}\right] .
$$

\section{MAXIMALLY COHERENT CP MAPS}

For a set of Kraus operators $\left\{\lambda_{i}\right\}$ defining a CP map $\Lambda$, we may define a measure of its self-coherence by inserting two independent instances of $\Lambda$ into both arms of an interferometer. If the CP map is unitary, then the interference pattern will have unit visibility. However, if there are more than one Kraus operator,

$$
\mathrm{v}=\frac{1}{d} \operatorname{Tr}\left[\lambda_{0}^{\dagger} \lambda_{0}\right]<1
$$

we can take this to measure the distance of $\Lambda$ to the set of unitaries.

It is interesting to ask, for a given $\Lambda$, and for all possible compatible sets of Kraus operators, what is the maximum visibility or self-coherence? In other words, for all sets of Kraus operators $\left\{\lambda_{i}\right\}$ implementing $\Lambda$, what is the largest value of $\frac{1}{d} \operatorname{Tr}\left[\lambda_{0}^{\dagger} \lambda_{0}\right]$ ? The canonical method of constructing a set of Kraus operators of a CP map is given by Choi [15]. The operators $\left\{\lambda_{i}\right\}$ created are linearly independent and thus represent the minimum number of operators required to represent $\Lambda$. If $\left\{\lambda_{i}^{\prime}\right\}$ also implement $\Lambda$, they are related by

$$
\lambda_{i}^{\prime}=\sum_{k} u_{i k} \lambda_{k}
$$

where $u$ is an isometry in general, or unitary when the number of elements in each set are equal. It can easily be shown that the largest possible visibility is obtained when $\left\{\lambda_{i}\right\}$ are orthogonal,

$$
\operatorname{Tr}\left[\lambda_{i}^{\dagger} \lambda_{j}\right]=\delta_{i j} \operatorname{Tr}\left[\lambda_{j}^{\dagger} \lambda_{j}\right],
$$


and the largest element is $\lambda_{0}$. When $\Lambda$ is placed in both arms, this upper limit is a measure of the intrinsic (de)coherence of the process. The visibility of an actual realisation of $\Lambda$ may be smaller than this maximum due to processes as in Eq. (18) but this does not represent intrinsic decoherence of the map itself.

We can also find the closest unitary operator to a given set of Kraus operators $\left\{\lambda_{i}\right\}$ which induce the CP map $\Lambda$ by considering an interferometer with $\Lambda$ occurring one arm, and a unitary $U$ operation in the other arm which we may alter as we like. We can maximise the interference pattern by changing $U$, and hence obtain the closest unitary to $\left\{\lambda_{i}\right\}$. The visibility is given by

$$
\mathrm{v}=\frac{1}{d}\left|\operatorname{Tr}\left[\lambda_{0}^{\dagger} U\right]\right|=\frac{1}{d}\left|\operatorname{Tr}\left[\sqrt{\lambda_{0} \lambda_{0}^{\dagger}} U_{\lambda_{0}} U\right]\right|
$$

where we have used the polar decomposition of $\lambda_{0}=$ $\sqrt{\lambda_{0} \lambda_{0}^{\dagger}} U_{\lambda_{0}}$ [16]. The visibility is thus maximised when $U=U_{\lambda_{0}}^{\dagger}$. If the eigenvalues of $\lambda_{0}^{\dagger} \lambda_{0}$ are $\left\{r_{j}\right\}$, then the visibility when $\Lambda$ is in both arms is simply $\mathrm{v}_{\Lambda \Lambda}=\sum r_{j}$, whereas if we compare $\Lambda$ with its closest unitary, it is $\mathrm{v}_{\Lambda U}=\sum \sqrt{r_{j}}$, and it is easy to see that $\mathrm{v}_{\Lambda U} \geq \mathrm{v}_{\Lambda \Lambda}$.

We can also consider what is the maximum coherent fidelity between two $\mathrm{CP}$ maps $\mathcal{U}$ and $\mathcal{V}$. Let $\left\{v_{i}\right\}$ and $\left\{\nu_{j}\right\}$ be orthogonal Kraus sets for $\mathcal{U}$ and $\mathcal{V}$ respectively (Eq. (24) $)$, and $\left(A_{i j}\right)=\left(\operatorname{Tr}\left[v_{i}^{\dagger} \nu_{j}\right]\right)$ be the matrix of their inner products. If $\left\{v_{i}^{\prime}=\sum_{j} g_{i j} v_{j}\right\}$ and $\left\{\nu_{i}^{\prime}=\sum_{j} h_{i j} \nu_{j}\right\}$ are also compatible Kraus operators for $\mathcal{U}$ and $\mathcal{V}$ (Eq. (23) $)$, then

$$
\left|\operatorname{Tr}\left[v_{0}^{\prime \dagger} \nu_{0}^{\prime}\right]\right|=\left|\sum_{i j} \operatorname{Tr}\left[g_{0 i}^{*} v_{i}^{\dagger} h_{0 j} \nu_{j}\right]\right|=\left|\sum_{i} g_{0 i}^{*} \sum_{j} A_{i j} h_{0 j}\right|
$$

is maximised when

$$
\begin{aligned}
\left\|A \vec{g}_{0}\right\| & =\max _{\|\vec{g}\|=1}\|A \vec{g}\| \\
\vec{h}_{0} & =\frac{A \vec{g}_{0}}{\left\|A \vec{g}_{0}\right\|},
\end{aligned}
$$

where we have used the operator norm of $A=\left(A_{i j}\right)$, and $\vec{g}_{0}$ and $\vec{h}_{0}$ are the first column vectors of the isometries $g_{i j}$ and $h_{i j}$ relating $\left\{v_{i}\right\}$ and $\left\{\nu_{j}\right\}$ to $\left\{v_{i}^{\prime}\right\}$ and $\left\{\nu_{i}^{\prime}\right\}$ respectively. This reduces to the previous case where both CP maps are the same and thus $\left(A_{i j}\right)$ is real diagonal.

\section{CONCLUSION}

Interferometry can be applied to the case of nonunitary processes to extract information about the un- derlying physical processes which implement them. In particular, we can derive a measure of the coherence of a quantum operation, its maximum for any CP map, and the closest unitary under this measure. It is to seen whether consideration of dynamical CP maps can impose further internal structure on quantum operations.

I would like to acknowledge the support of the Cambridge-MIT Institute Quantum Information Initiative, EU grants RESQ (IST-2001-37559) and TOPQIP (IST-2001-39215). I also thank S. G. Schirmer, A. K. Ekert, A. P. A. Kent, A. Landahl, B-G. Englert, D. Kaszlikowski, L. C. Kwek, A. J. Short and B. M. J. B. D. Walker for helpful discussions.

[1] M. A. Nielsen, I. L. Chuang, Quantum Computation and Quantum Information, Cambridge University Press (2000)

[2] K. Kraus, States, Effects, and Operations, SpringerVerlag, Berlin (1983)

[3] J. Åberg, electronic pre-print quant-ph/0302182

[4] A. M. Childs, I. L. Chuang, D. W. Leung, Realization of Quantum Process Tomography in NMR, Phys. Rev. A. 64, art. no. 012314 (2001)

[5] P. D. D. Schwindt, P. K. Kwiat, B-G. Englert, Quantitive wave-particle duality and non-erasing quantum erasure, Phys. Rev. A 60, p4285 (1999)

[6] S. Pancharatnam, Proc. Indian Acad. Sc. 44, p247 (1956)

[7] A. K. Ekert, C. Moura Alves, D. K. L Oi, M. Horodecki, P. Horodecki, L. C. Kwek, Direct Estimations of Linear and Nonlinear Functionals of a Quantum State, Phys. Rev. Lett. 88, art. no. 217901 (2002)

[8] D. K. L. Oi, D. Angelakis, R. Rodriquez, B. Falabretti, Forthcoming

[9] W. F. Stinespring, Positive Maps on $\mathrm{C}^{*}$-Algebras, Proc. Amer. Math. Soc. 6, p211 (1955)

[10] M. Ericsson, E. Sjöqvist, J. Brännlund, D. K. L. Oi, A. K. Pati, quant-ph/0205160

[11] J. G. Peixoto de Faria, A. F. R. de Toledo Piza, M. C. Nemes, quant-ph/0205146

[12] A. Jamiołkowski, Rep. Math. Phys. 3, p275 (1972)

[13] A. Uhlmann, The transition probability in the state space of a *-algebra, Rep. Math. Phys. 9, p273 (1976)

[14] M. Raginsky, A Fidelity Measure for Quantum Channels, Phys. Lett. A 290, p11 (2001)

[15] M. D. Choi, Linear Algebra and its Applications 10, p285 (1975)

[16] R. A. Horn, C. R. Johnson, Matrix Analysis, Cambridge University Press (1985)

[17] Thanks to B-G Englert for pointing out this subtle case. 